\title{
Ectopic thyroid tissue presenting as a cardiac mass
}

\author{
Steven Henderson ${ }^{1}$, Adam Din $^{2}$, Wael Elsaify ${ }^{3}$ \\ Sri Lanka Journal of Diabetes, Endocrinology and Metabolism 2013; 3: 98-100
}

\begin{abstract}
Thyroid ectopia is a rare, congenital phenomenon. Ectopic thyroid tissue located within the heart is extremely rare, with less than 30 cases ever reported. We present one such case with, what we believe, is a unique presentation.

A 46 year old woman presented with chest pain. Echocardiogram demonstrated a right ventricular mass which was later excised and found to be thyroid tissue. Multiple investigations failed to demonstrate any further abnormality and a diagnosis of intra-cardiac thyroid ectopia was made.

A variety of embryological abnormalities can lead to thyroid ectopia and it is linked to several genetic mutations. Interruption of thyroid migration can lead to thyroid tissue being deposited anywhere along its path. Before the diagnosis of ectopia is made, however, thyroid malignancy must first be excluded. Other differential diagnoses include struma ovarii and malignant emboli. Although exceptionally rare, intra-cardiac thyroid ectopia should be considered for any unusual cardiac mass prior to invasive treatment or investigation.
\end{abstract}

Key words: thyroid, ectopia, cardiac.

\section{Introduction}

Primary cardiac tumours are rare, usually benign lesions with an overall incidence of $0.05-0.5 \%(1,2)$. The most common form of primary cardiac tumour, accounting for $25 \%$ of cases, is a myxoma, a pedunculated tumour arising from the endocardium (3). More commonly, cardiac tumours are metastatic deposits, although these are still rare with an incidence of less than $1 \%$ of cancer patients (1).

Thyroid ectopia is defined as thyroid tissue at any site other than anterolateral to tracheal rings 2-4 $(1,4,5)$. Again, it is a rare congenital phenomenon occurring in 1 per 100,000-300,000 of the general population (5). Ectopic intra cardiac thyroid tissue is rarer still, with less than 30 cases reported worldwide in the literature since it was first described in 1941 by Dosch (6-9).

Despite this, it is an important differential in the diagnosis of a cardiac mass. We present one such case in which the patient presented with chest pain followed by a brief review of the available literature.

\section{Case report}

A 46 year old woman, with a background of noninsulin dependent diabetes, presented with an 8 week history of left sided chest pain. The pain was dull in nature, did not radiate and had no other associated features. It was not related to exercise and, by the time of her presentation, was occurring daily. She had previously undergone hysterectomy and appendicectomy. There was no family history of note and she was a lifelong smoker.

In the first instance, an ECG and echocardiogram were arranged. The ECG showed normal sinus rhythm with no evidence of ischaemic injury. The echocardiogram demonstrated a $2.6 \times 1.6 \mathrm{~cm}$ right ventricular mass (Figure 1 ). Ventricular size and function were normal and there was no valve dysfunction. Subsequent CT pulmonary angiogram did not demonstrate any pulmonary lesions although an incidental left breast fibroadenoma was detected. Completion CT abdomen and pelvis showed no other lesion. The ovaries were noted to be cystic and the thyroid was unremarkable. A bone scan was also reported as normal. Prior to surgery, the patient underwent coronary angiogram which demonstrated only mild disease of the

${ }^{1}$ Department of Radiology, Victoria Infirmary, Glasgow, ${ }^{2,}{ }^{3}$ Department of Endocrine Surgery, James Cook University Hospital, Middlesbrough, UK. 
left anterior descending artery (LAD). The mass in the right ventricle derived its blood supply from the septal branch of the LAD.

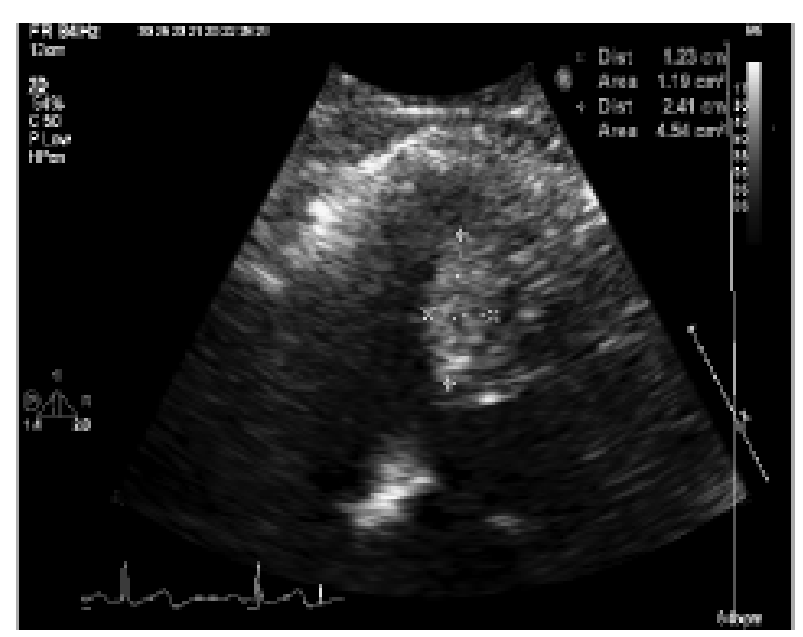

Figure 1. Echocardiogram of cardiac mass.

The patient underwent surgical resection and a well circumscribed $2.4 \times 1.9 \times 1.8 \mathrm{~cm} 6$ g nodule was excised. Histologically, the sample was described as well-defined thyroid tissue containing varied size follicles and no features to suggest malignancy. Differential diagnosis included metastatic follicular carcinoma of the thyroid gland, metastatic struma ovarii or ectopic thyroid tissue.

Following this, ultrasound of the pelvis was performed although this demonstrated only bilateral cystic ovaries as previously demonstrated on CT. An ultrasound of the neck showed an essentially normal thyroid gland with several small colloid cysts in both lobes. Thyroid function tests were normal.

Following multidisciplinary team review and postoperative whole body CT scan, the diagnosis of intracardiac ectopic thyroid tissue was made.

\section{Discussion}

In normal human embryology, the thyroid gland develops at around day 24 of gestation (10). The thyroid gland derives from a midline thickened out pouching of the endodermal epithelium of the pharynx between pharyngeal pouches 1 and 2, directly dorsal to the aortic sac $(2,11)$. In the early stages, the thyroid is in close proximity to the bulbus cordis, from where the right heart develops. All but 1 case of intracardiac thyroid ectopia reported in the literature have originated in the right heart (12). This has led to the hypothesis that abnormal persistent contact between the two structures may be implicated in pathogenesis $(1,6,8,9)$.
The thyroid is connected to the pharyngeal floor by the thyroglossal duct in the early stages of development but this normally disappears by week 10 . The thyroid descends along the path of the thyroglossal duct in front of the trachea, anterior to the hyoid bone and thyroid cartilage, to its final pre-tracheal position in gestational week $7(1,5,10)$. Interruption of this descent is the most common cause for thyroid ectopia (13). As a result, lingual thyroid is the most frequent, occurring in $90 \%$ of cases. Surpahyoid, infrahyoid, submandibular, tracheal, lateral cervical, axillary, palatine tonsils and the bifurcation of the carotid artery are all locations in the head and neck region that have been reported to contain ectopic thyroid tissue $(2,5)$. Ectopic thyroid tissue has also been reported at more distant sites in the liver, duodenum, jejunum, pancreas, porta hepatis, gallbladder, stomach, adrenal gland, aorta and oesophagus $(4,5,10)$ explained, most likely, by over-descent of the thyroid during embryogenesis.

Several genetic mutations are postulated to be associated with thyroid ectopia. Titf1, Foxe1, Pax 8, Hhex and Tshr are required for the normal development of the thyroid gland. Animal studies have suggested that genetic abnormalities in these genes could be associated with abnormal thyroid development and possible thyroid ectopia $(5,14)$.

Once the presence of thyroid tissue is confirmed in the cardiac tissue, it is important to exclude metastatic follicular carcinoma. Thyroid carcinoma metastasising to the heart is rare, occurring in less than $1 \%$ of cases $(1,9)$. Metastatic thrombi from a primary thyroid cancer can be deposited in the heart via the thyroid veins (9).

Struma ovarii, another important differential, is an ovarian teratoma that consists of mature thyroid epithelium $(15,16) .15 \%$ of cases of struma ovarii may manifest hyperthyroid features and, importantly, up to 5\% may develop carcinoma (15).

The clinical presentation of cardiac tumours is often non-specific and usually does not cause symptoms until cardiac function is impaired $(1,6)$. Palpitations/arrhythmias, dizzy spells, conduction defects and emboli can, however, be features $(1,6,8,9,12)$. We believe our patient to be the first to present with isolated chest pain.

Cardiac masses are most commonly identified on an echocardiogram. Once the presence of a cardiac mass is confirmed, further investigation with CT or MRI is common $(1,2,7)$. Coronary angiography will identify the blood supply to the tumour and help with surgical planning (1). Overall, the available literature reports generally good results from surgical resection $(7,10,13)$. Once the mass is identified as thyroid tissue, the thyroid must be investigated for evidence of primary malignancy. 
It is important to note that, as ectopic thyroid tissue contains mature thyroid epithelium, it can produce thyrotoxicosis and can be a source of a primary thyroid carcinoma (5).

\section{Conclusion}

Ectopic, intracardiac thyroid tissue is a rare cause of a cardiac mass. It is vital that metastatic thyroid carcinoma is excluded following confirmation of intracardiac thyroid tissue.

\section{References}

1. Comajuan S, Ayerbe J, Ferrer B, Quer C, et al. An intracardiac ectopic thyroid mass. European Journal of Echocardiography 2009; 10: 704-6.

2. Altay C, Erdogan N, Karasu S, Uluc E, et al. CT and MRI findings of developmental abnormalities and ectopia varieties of the thyroid gland. Diagn Interv Radiol 2012; 18: $335-43$

3. Underwood JCE. General and Systematic Pathology ISBN 0443073341.

4. Salam M, Mohideen A, Stravitz R. Ectopic thyroid presenting as a liver mass. Clinical Gastroenterology and Hepatology 2012; 10: 30.

5. Ibrahim N, Fadeyibi I. Ectopic thyroid: etiology, pathology and management. Hormones 2011; 10(4): 261-9.

6. Polvani G, Antonia C, Porqueddu M, Pompilio G, Cavoretto
D, et al. Intracardiac ectopic thyroid: Conservative surgical treatment. Ann Thorac Surg 1993; 55: 1249-51.

7. Wu Z, Zhou Q, Wang D. An intracardiac ectopic thyroid adenoma. Interactive CardioVascular and Thoracic Surgery 2009; 8: 587-8.

8. Richmond I, Whittaker J, Deiraniya A, Hassan R. Intracardiac ectopic thyroid: a case report and review of published cases. Thorax 1990; 45: 293-4.

9. Hirnle T, Szymczak J, Ziolkowski P, Lenartowska L. European Journal of Cardio-thoracic Surgery 1997; 12: 147-9.

10. Mace A, Taghi A, Khalil S, SandisonA. Ectopic sequestered thyroid tissue: An unusual cause of a mediastinal mass. ISRN Surg 2011: 313626.

11. Gray’s Anatomy 39th Edition ISBN 0443071683.

12. Landeta F, Hoffmeier A, Fuchs M, Scheld H, et al Ectopic thyroid mass in the heart. Lancet 2012: 379: 1762.

13. Chataigner O, Mussot S, Fadel E, Dartvelle P. Two cases of intra-pericardial tumours arising from the ascending aorta in adults. European Journal of Cardio-thoracic Surgery 2007; 32:174-5.

14. Heather N, Hall K, Neas K, Potter H, et al. Growth and development in a child with resistance to thyroid hormone and ectopic. Thyroid Gland Paediatrics 2012; 129: e817.

15. Bozkurt N, Karbeck B, Ozkaya E, Cakal E, et al. J Med Case Reports 2011; 5: 572.

16. Essential Obstetrics and Gynaecology 4th Edition ISBN 0443071470. 